\title{
Extensional and Flexural Waves in a Thin-Walled Graphite/Epoxy Tube*
}

\author{
William H. Prosser \\ NASA Langley Research Center \\ Hampton, VA 23665 \\ Michael R. Gorman \\ Aeronautics and Astronautics \\ Naval Postgraduate School \\ Monterey, CA 93943 \\ John Dorighi** \\ NASA Langley Research Center \\ Hampton, VA 23665 \\ Journal of Composite Materials \\ Vol. 26(14), 1992, pp. 418-427
}

\begin{abstract}
Simulated acoustic emission signals were induced in a thinwalled graphite/epoxy tube by means of lead breaks (Hsu-Neilsen source). The tube is of similar material and layup to be used by NASA in fabricating the struts of space station Freedom. The resulting waveforms were detected by broad band ultrasonic transducers and digitized. Measurements of the velocities of the extensional and flexural modes were made for propagation directions along the tube axis (0 degrees), around the tube circumference (90 degrees) and at an angle of 45 degrees. These velocities were found to be in agreement with classical plate theory.

* Work supported by NASA Langley Research Center

** Mechanical Engineering student, University of Colorado, Boulder, CO 80309-0427
\end{abstract}




\section{Introduction}

Graphite/epoxy composites, because of their high strength, high stiffness, and light weight, have been chosen for fabrication of the strut tubes of Space Station Freedom (SSF). While exposed to the harsh environment of space, these tubes will be subjected to hypervelocity micrometeriod impacts and large thermal cycling, among other things. Thus, some method of monitoring these tubes is needed while SSF is in orbit. Other critical parts which need to be monitored include the various fiber wrapped pressure vessels used for life support and fuel containment purposes.

Acoustic emission ( $\mathrm{AE}$ ) testing has been proposed as a technique for nondestructively monitoring these structures. The global monitoring capability of $\mathrm{AE}$ makes it well suited for this purpose. However, much information about the generation and propagation of acoustic emission signals in composites and composite tubes is needed before AE techniques can yield useful quantitative information.

Gorman and Ziola [1] demonstrated that simulated AE signals in flat composite plates consist of the lowest order Lamb modes. These modes are often referred to as plate modes. The lowest order symmetric mode reduces to what is called the extensional mode in plate theory, while the lowest order anti-symmetric mode reduces to the flexural mode; these terms apply when the plate is thin, that is, the wavelength is much greater than the thickness. The signals were generated by pencil lead breaks (also known as Hsu-Neilsen sources). Gorman and Ziola [1] further showed that real AE signals generated by transverse matrix cracking in a composite propagate as plate modes.

The propagation of Lamb and plate modes in composite plates has been studied by numerous investigators. Noiret and Roget [2] investigated the case of long wavelength and low frequencies. Chimenti and Nayfeh [3, 4] have extensively studied Lamb mode propagation for ultrasonic materials characterization measurements. Veidt and Sayir [5] also characterized the material 
properties by measuring the flexural plate mode velocity. Stiffler and Henneke [6] investigated plate modes in an attempt to better understand the acousto-ultrasonic technique. A number of investigators have studied plate waves generated by the impact of a composite including Moon [7], Chow [8], Sun and Lai [9], and Rose and Mortimer [10].

With the exception of the study by Rose and Mortimer [10] who also studied composite shells or tubes, the previous research has all focussed on the simple geometry of a flat plate. The majority of practical structures, such as the strut tubes studied in this research, are of more complicated geometries. This research demonstrates, however, that AE signals propagate in these thinwalled tubes as plate waves and that classical plate theory is adequate for the prediction of their propagation velocities.

Gorman and Ziola [1] further pointed out that an understanding of the propagation of plate modes with different velocities is needed to improve the location capability of AE techniques. They also investigated the effect of source orientation by breaking pencil leads on the surface and on the edge of the plate. An interesting feature of these plate modes was that the amplitude of the displacement components of the different modes was dependent on the source orientation for the simulated AE sources. For the composite plates, if the lead was broken on the surface, the resulting waveform had a large out-of-plane component for the flexural mode and a much smaller out-of-plane component for the extensional mode. For lead breaks on the edge of the plate, the out-of-plane component of the extensional mode was much larger than that of the flexural mode. Gorman [11] showed similar results on aluminum plates for surface and edge breaks.

Gorman and Prosser [12] additionally demonstrated that the amplitudes of the displacement components of the extensional and flexural modes were dependent on source orientation for intermediate angles on aluminum plates. This was accomplished by machining angled slots into the plate on which the lead was broken. This feature of plate waves may be useful in determining the type of source and its orientation for actual AE signals. For 
example, the observed out-of-plane displacement components of plate waves due to transverse matrix cracking in the work of Gorman and Ziola [1] had extensional amplitudes which were much larger than the flexural. This makes sense since transverse matrix cracking should produce a source motion in the plane of the plate generating a larger extensional wave.

In this research, the out-of-plane components of $\mathrm{AE}$ signals produced by lead breaks on a graphite/epoxy tube of dimensions and design to be used on SSF were measured using broad band transducers. The signals were shown to consist of plate modes. The amplitudes of the modes were again shown to be affected by source orientation by creating lead breaks on the edge as well as the surface of the tube. Since the specimen being tested is actually a tube geometry, the plate wave predictions are only an approximation. However good agreement might be expected at the frequencies measured since the wavelengths are smaller than the radius of curvature. Measurements of the velocities of both modes were made for propagation along the tube (0 degrees), around the tube circumference (90 degrees), and at 45 degrees. These velocities were then compared with velocities predicted from classical plated theory using stiffness coefficients predicted by laminated plate theory and good agreement was demonstrated.

\section{Theory}

Classical plate theory predicts three modes of propagation in a plate [13]. These are called the extensional, the in-plane shear and the flexural modes. This theory is based on the assumption of plane stress in a thin plate where the wavelength is large in comparison with the plate thickness. The in-plane shear mode is not detectable when using a transducer which is sensitive to outof-plane displacement and is mounted on one of the faces of the plate. Thus, it was not observed previously by Gorman and ziola [1] and has not been detected in this research. Therefore, the theoretical treatment of this mode is not presented here. 
While classical theory predicts only an in-plane displacement component for the extensional mode, higher order theories predict [14, 15] and experimental measurements verify the existence of an out-of-plane displacement component for this mode. This out-ofplane component is due to the Poisson effect from the in-plane motion and thus propagates at the same velocity. In a homogeneous isotropic material the extensional mode propagates with a velocity $c_{e}$ which is dispersionless:

$$
c_{e}=\sqrt{\frac{E}{\rho\left(1-v^{2}\right)}}
$$

where $E$ is the Young's modulus, $v$ is Poisson's ratio, and $\rho$ is the density.

In anisotropic composite materials the extensional velocity is dependent on the in-plane stiffness coefficients $A_{i j}, i, j=1,2$, and 6 in the usual contracted notation. These are referred to the laminate axes, $x$ and $y$, which are in the plane of the plate. The $A_{i j}$ are defined as

$$
A_{i j}=\int_{z=-\frac{h}{2}}^{z=\frac{h}{2}} Q_{i j}^{(k)} d z
$$

where $\mathrm{h}$ is the thickness of the laminate, $\mathrm{z}$ is the distance in the thickness direction from the midplane of the laminate, and $Q_{i j}^{(k)}$ are the lamina stiffness coefficients for the $\mathrm{k}^{\text {th }}$ lamina of the composite. For a +/- angle-ply symmetric laminate, only four of the in-plane stiffness coefficients are non-zero. These are $\mathrm{A}_{11}$, $A_{12}, A_{22}$, and $A_{66}$. The equations of motion for the in-plane displacements for a material with this symmetry are given by Whitney [16] as 


$$
A_{11} \frac{\partial^{2} u}{\partial x^{2}}+A_{66} \frac{\partial^{2} u}{\partial y^{2}}+\left(A_{12}+A_{66}\right) \frac{\partial^{2} v}{\partial x \partial y}=\rho h \frac{\partial^{2} u}{\partial t^{2}}
$$

and

$$
A_{66} \frac{\partial^{2} v}{\partial x^{2}}+A_{22} \frac{\partial^{2} v}{\partial y^{2}}+\left(A_{12}+A_{66}\right) \frac{\partial^{2} u}{\partial x \partial y}=\rho h \frac{\partial^{2} v}{\partial t^{2}}
$$

where $\mathrm{u}$ is the displacement in the $\mathrm{x}$ direction and $\mathrm{v}$ is the displacement in the y direction.

These equations govern the propagation of the extensional and the in-plane shear modes. Except along symmetry directions of the composite, neither mode will be pure extensional or pure shear. Instead, they will be quasi-extensional or quasi-shear modes. The mode with its largest component of particle displacement in the direction of propagation is the quasi-extensional mode.

The dispersion behavior of the in-plane modes is obtained by substituting the displacements for a plane wave propagating in an arbitrary direction into the equations of motion. The displacements are

$$
u=A_{0} \alpha_{x} e^{i\left(\omega t-k I_{x} x-k l_{y}\right)}
$$

and

$$
V=A_{0} \alpha_{y} e^{i\left(\omega t-k l_{x} x-k l_{y Y}\right)}
$$

where $A_{0}$ is the amplitude, $\alpha_{i}$ are the direction cosines of the particle displacements, $\omega$ is the angular frequency, $k$ is the wavenumber, and $l_{i}$ are the direction cosines of the wave normal. Substitution of the displacements and reducing terms yields

$$
\left[\begin{array}{cc}
\mathrm{A}_{11} l_{\mathrm{x}}^{2}+\mathrm{A}_{66} \mathrm{l}_{\mathrm{y}}^{2}-\rho h \mathrm{c}^{2} & \left(\mathrm{~A}_{12}+\mathrm{A}_{66}\right) \mathrm{l}_{\mathrm{x}} \mathrm{l}_{\mathrm{y}} \\
\left(\mathrm{A}_{12}+\mathrm{A}_{66}\right) \mathrm{l}_{\mathrm{x}} \mathrm{l}_{\mathrm{y}} & \mathrm{A}_{66} \mathrm{l}_{\mathrm{x}}^{2}+\mathrm{A}_{22} \mathrm{l}_{\mathrm{y}}^{2}-\rho h \mathrm{c}^{2}
\end{array}\right]\left[\begin{array}{l}
\alpha_{\mathrm{x}} \\
\alpha_{\mathrm{y}}
\end{array}\right]=0
$$


where $\mathrm{c}$ is the phase velocity and is equal to $\omega / \mathrm{k}$. The nontrivial solution for this equation occurs when the determinant of the coefficients is equal to zero which yields a quadratic in $\mathrm{phc}^{2}$. The solution which predicts the faster velocity is that for the quasi-extensional mode.

For propagation along the $\mathrm{x}$ axis, the extensional mode is a pure mode wave and its velocity is given by

$$
c_{e}=\sqrt{\frac{A_{11}}{\rho h}} .
$$

The extensional mode is also pure mode for propagation along the $y$ direction with the velocity given by

$$
c_{e}=\sqrt{\frac{A_{22}}{\rho h}} .
$$

For propagation at 45 degrees between the $x$ and $y$ axes, the solution yields a quasi-extensional mode with a velocity given by

$$
C_{e}=\sqrt{\frac{\left(A_{11}+2 A_{66}+A_{22}+\sqrt{R}\right.}{4 \rho h}}
$$

where

$$
R=\left(A_{11}+2 A_{66}+A_{22}\right)^{2}-4\left(A_{11}+A_{66}\right)\left(A_{22}+A_{66}\right)+4\left(A_{12}+A_{66}\right)^{2}
$$

The second mode of propagation is the flexural mode. For isotropic materials, this mode has a velocity $c_{f}$ which is given by

$$
C_{f}=\sqrt[4]{\frac{D}{\rho h}} \sqrt{\omega}
$$

where $\mathrm{D}$ is the bending stiffness defined by 


$$
\mathrm{D}=\frac{\mathrm{Eh}^{3}}{12\left(1-v^{2}\right)}
$$

and $\omega$ is the frequency in radians/sec. This mode is dispersive with the higher frequencies traveling at higher velocities.

For laminated composite materials, the flexural velocity is a function of the bending stiffness coefficients $\left(D_{i j}\right)$. These are defined as

$$
D_{i j}=\int_{z=-\frac{h}{2}}^{z=\frac{h}{2}} Q_{i j}^{(k)} z^{2} d z
$$

In a symmetric angle-ply composite, the six coefficients $D_{11}, D_{12}$, $\mathrm{D}_{16}, \mathrm{D}_{22}, \mathrm{D}_{26}$, and $\mathrm{D}_{66}$ are nonzero. The equation of motion is given by

$$
\begin{gathered}
D_{11} \frac{\partial^{4} w}{\partial x^{4}}+4 D_{16} \frac{\partial^{4} w}{\partial x^{3} \partial y}+2\left(D_{12}+D_{66}\right) \frac{\partial^{4} w}{\partial x^{2} \partial y^{2}} \\
+4 D_{26} \frac{\partial^{4} w}{\partial x \partial y^{3}}+D_{22} \frac{\partial^{4} w}{\partial y^{4}}=-\rho h \frac{\partial^{2} w}{\partial t^{2}}
\end{gathered}
$$

where $w$ is the out-of-plane displacement. The dispersion relation is again obtained by assuming a plane wave of the form

$$
w=A_{0} e^{i\left(\omega t-k l_{X X}-k l_{y} Y\right)}
$$

Again, the velocity is dispersive and is dependent on the propagation direction. It is given by

$$
c_{f}=\sqrt[4]{\frac{D_{11}^{\prime}}{\rho h}} \sqrt{\omega}
$$

where 


$$
D_{11}^{\prime}=D_{11} I_{x}^{4}+D_{22} I_{y}^{4}+2 D_{12} I_{x}^{2} I_{y}^{2}+4 D_{66} I_{x}^{2} I_{y}^{2}+4 D_{16} I_{x}^{3} I_{y}+4 D_{26} I_{x} I_{y}^{3}
$$

\section{Experiment}

The tube studied in this research was AS4/976 graphite/epoxy with a laminate stacking sequence of $[+/-10,+/-30,+/-10] \mathrm{s}$. The tube had dimensions of 4.5 meters in length, $0.0508 \mathrm{~m}$. in inner diameter, and $0.0035 \mathrm{~m}$. in wall thickness.

Pencil lead breaks were used to simulate acoustic emission signals in the tube. The waves were detected using a $3.5 \mathrm{MHz}$ ultrasonic transducer (Panametrics) with a diameter of $1.27 \mathrm{~cm}$. This transducer was used because of its flat frequency response in the 20 to $500 \mathrm{kHz}$ bandwidth of the observed signals. The detected signals were amplified and filtered with a preamplifier (Physical Acoustics Corporation 1220A) which had a $20 \mathrm{kHz}$ high pass filter. The signals were then digitized by a transient recorder (LeCroy 6810) operating at a 5 MHz sampling rate with a 12 bit vertical resolution. The digitized signals were stored on a computer for later analysis. Figure 1 shows a block diagram of the experimental setup. A typical waveform at a source to receiver distance of $0.1524 \mathrm{~m}$. is shown in Figure 2 wherein the extensional and flexural waveform components are identified. The waveforms at other source to receiver distances are similar with the extensional mode arriving earlier because of its faster velocity. The time between the extensional and flexural mode arrivals increases with increasing distance of propagation because of the slower velocity of the flexural mode.

Measurements of the extensional and flexural velocities were made along the tube axis (0 degrees or $x$-direction), around the tube circumference (90 degrees or y-direction), and at an angle of 45 degrees to the axis of the tube. For measurement of the velocity along a given direction, the transient recorder was triggered by a sensor placed next to the source and the arrival 
times of the extensional and flexural modes were recorded for a given distance of propagation. The receiving transducer was then moved by a preselected distance along the desired propagation direction and the lead break repeated. Four to six points data were taken for each mode and direction of propagation. The values of arrival time were then plotted against the trigger sensor to receiving sensor separation. A linear least squares fit was applied to the data and the slope was taken to be the velocity.

For the extensional wave, the earliest detected zero crossing was used for the arrival time. However, because the flexural mode is dispersive, the arrival time must be measured at a particular frequency to allow a comparison with theory. In addition, classical plate theory agrees with exact theory only at low frequencies. The lowest frequency in the data which was unperturbed by refections was at about $50 \mathrm{kHz}$. As a rough measurement of the arrival time of this frequency, the time for the arrival of the portion of the wave with a half cycle time of 10 usec. was used. This was used to compute the velocity.

Signals were also obtained when the pencil lead was broken on the end of the tube. In this case the source motion was primarily in-plane. This was done so that the out-of-plane displacement components of the extensional and flexural motion could be compared with those where the source motion was normal to the plane (surface breaks).

\section{Results and Discussion}

Table 1 shows the material properties of the lamina obtained from the manufacturer's data sheet. Also in Table 1 are the laminated plate theory calculations for the in-plane and bending stiffness coefficients for a laminated plate with the same stacking sequence as that of the tube.

The theoretical extensional and flexural velocity predictions are given in Table 2 for the three different directions of 
propagation. Because of the dispersive nature of the flexural mode, its velocity prediction is expressed in terms of the frequency of the wave.

The arrival time versus distance plot for extensional waves propagating along the 0 degree direction is shown in Figure 3 . The dotted line is a least squares fit to the data. The experimentally measured velocity values are also presented in Table 2. The arrival time versus distance plot for the 0 degree direction of the $50 \mathrm{kHz}$ component of the flexural wave is presented in Figure 4. A least squares fit is indicated by the dotted line. The theoretical velocity at this frequency and the experimental values are also given in Table 2. Good agreement was obtained between theory and experiment except for the 45 degree extensional mode and the 90 degree flexural mode, which need further investigation.

A waveform due to a lead break on the edge of the tube is presented in Figure 5. A comparison with Figure 2, which shows a waveform for an identical distance of propagation but with the lead break on the surface of the tube, demonstrates the effect of source orientation on the amplitudes of the plate waves. The edge break produces an extensional wave with a large out-of-plane displacement component. The surface break also produces a large out-of-plane displacement component, but in the flexural mode. As discussed previously, this fact may be useful in obtaining source information for actual sources in composites.

\section{Acknowledgements}

The authors would like to thank Mr. Charles Salkowski of the NASA Johnson Space Center for providing the tube specimen. The continuing support of this work by J. S. Heyman of the Nondestructive Measurement Science Branch of the NASA Langley 
Research Center is gratefully acknowledged. E. I. Madaras is the program monitor.

\section{References}

1. Gorman, M. R., and S. M. Ziola, "Plate Waves Produced by Transverse Matrix Cracking," Ultrasonics, (In Press).

2. Noiret, D. and J. Roget, "Calculation of Wave Propagation in Composite Materials Using the LAMB Wave Concept," Journal of Composite Materials, Vol. 23 (1989) pp. 195-206.

3. Chimenti, D. E., and A. H. Nayfeh, "Ultrasonic Reflection and Guided Wave Propagation in Biaxially Laminated Composite Plates," Journal of the Acoustical Society of America, Vol. 87 (1990) pp. 1409-1415.

4. Nayfeh, A. H., and D. E. Chimenti, "Propagation of Guided Waves in Fluid-Coupled Plates of Fiber-Reinforced Composite," Journal of the Acoustical Society of America, Vol. 83 (1988) pp. 1736-1743.

5. Veidt, M., and M. Sayir, "Experimental Evaluation of Global Composite Laminate Stiffnesses by Structural Wave Propagation," Journal of Composite Materials, Vol. 24 (1990) pp. 688-706.

6. Stiffler, R. C., and E. G. Henneke II, "Low Frequency Plate Wave Modes", NASA Contractor Report 3976 (1986), pp. II-1 II-31.

7. Moon, F. C., "Wave Surfaces Due to Impact on Anisotropic Plates," Journal of Composite Materials, Vol. 7 (1973) pp. 6279 .

8. Chow, T. S., "On the Propagation of Flexural Waves in an Orthotropic Laminated Plate and Its Response to an Impulsive 
Load," Journal of Composite Materials, Vol. 5 (1971) pp. 306319 .

9. Sun, C. T., and R. Y. S. Lai, "Exact and Approximate Analyses of Transient Wave Propagation in an Anisotropic Plate," AIAA Journal, Vol 12 (1974) pp. 1415-1417.

10. Rose, J. L., and R. W. Mortimer, "Wave Velocities in Unidirectional Graphite Epoxy Shells," Journal of Composite Materials, Vol. 8 (1974) pp. 191-194.

11. Gorman, M. R., "Plate Wave Acoustic Emission," Journal of the Acoustical Society of America (in press).

12. Gorman, M. R., and W. H. Prosser, "Acoustic Emission Source Orientation by Plate Wave Analysis," Journal of Acoustic Emission (in press).

13. Graff, K., Elastic Wave Motion in Solids, Ohio State University Press (1976).

14. Mindlin, R. D., and M. A. Medick, "Extensional Vibrations of Elastic Plates," Journal of Applied Mechanics, Vol. 26 (1959) pp. 561-569.

15. Kane, T. R., and R. D. Mindlin, "High-Frequency Extensional Vibrations of Plates, Journal of Applied Mechanics, Vol. 23 (1956) pp. 277-283.

16. Whitney, J. M., Structural Analysis of Laminated Anisotropic Plates, Technomic Publishing Co. (1987). 
Table 1. Properties of AS4/976 Single Lamina and Laminated Plate.

\section{Single Lamina}

AS4/976 (Fiberite)

\begin{tabular}{|c|c|c|c|c|c|}
\hline $\mathrm{E}_{\mathrm{x}} \quad(\mathrm{GPa})$ & $E_{y}(G P a)$ & $E_{S}(G P a)$ & $v_{x}$ & $v_{y}$ & $\rho\left(\mathrm{Kg} / \mathrm{m}^{3}\right)$ \\
\hline 147.6 & 9.65 & 3.2 & 0.30 & 0.02 & 1560 \\
\hline
\end{tabular}

\section{Laminated Plate Stiffness}

$[+/-10,+/-30,+/-10]_{S}$

Laminate thickness $\mathrm{h}=3.528 \times 10^{-3} \mathrm{~m}$

In-plane stiffness (MPa-m)

$\begin{array}{llll}\mathrm{A}_{11} & \mathrm{~A}_{22} & \mathrm{~A}_{12} & \mathrm{~A}_{66} \\ 433 & 44.6 & 50.8 & 51.6\end{array}$

Bending stiffness $\left(\mathrm{Pa}-\mathrm{m}^{3}\right)$

$\begin{array}{llllll}\mathrm{D}_{11} & \mathrm{D}_{22} & \mathrm{D}_{12} & \mathrm{D}_{66} & \mathrm{D}_{16} & \mathrm{D}_{26} \\ 463 & 43.9 & 46.7 & 47.6 & 27.9 & 4.96\end{array}$


Table 2. Theoretical and Experimental Plate Wave Velocities

Extensional Waves

$\begin{array}{ccc}\text { Angle } & \text { Theoretical } \mathrm{c}_{e} & \text { Experimental } \mathrm{c}_{e} \\ \text { (degrees) } & (\mathrm{km} / \mathrm{s}) & (\mathrm{km} / \mathrm{s}) \\ 0 & 8.87 & 8.89 \\ 45 & 5.97 & 4.13 \\ 90 & 2.85 & 3.12\end{array}$

\section{Flexural Waves}

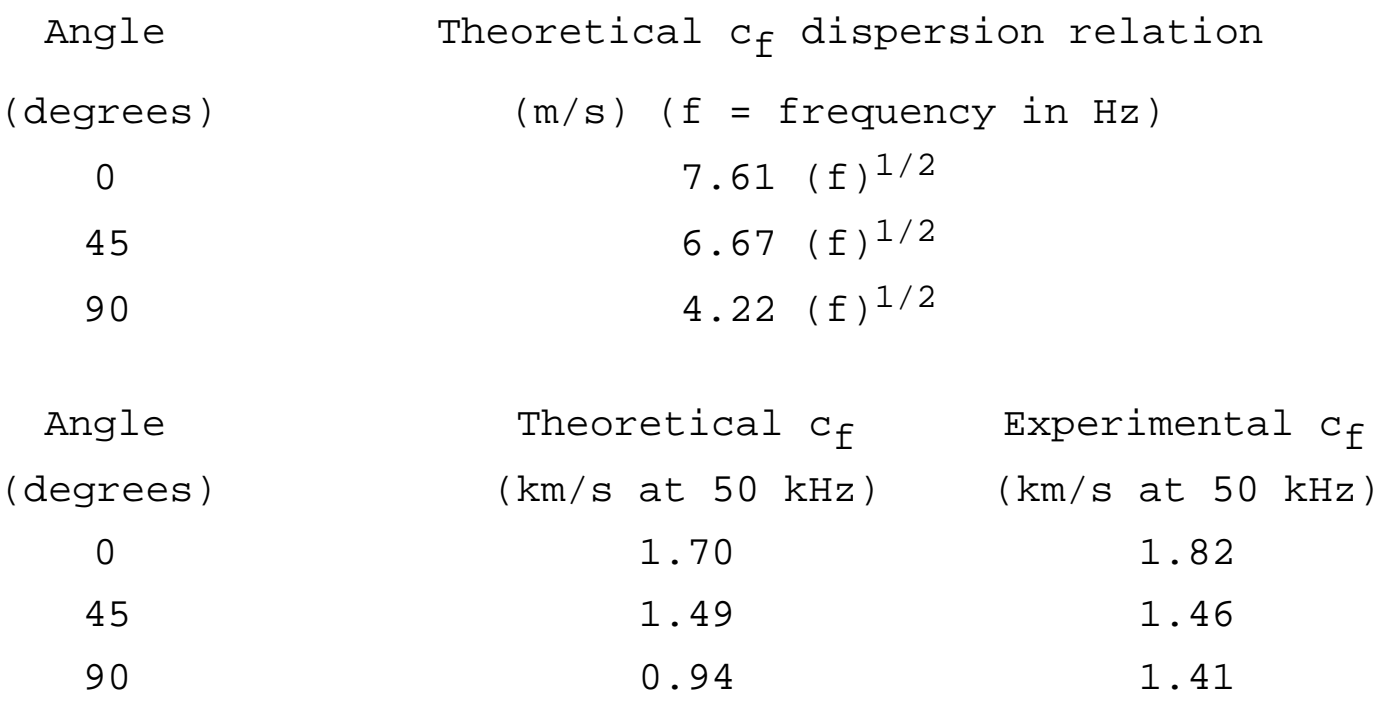




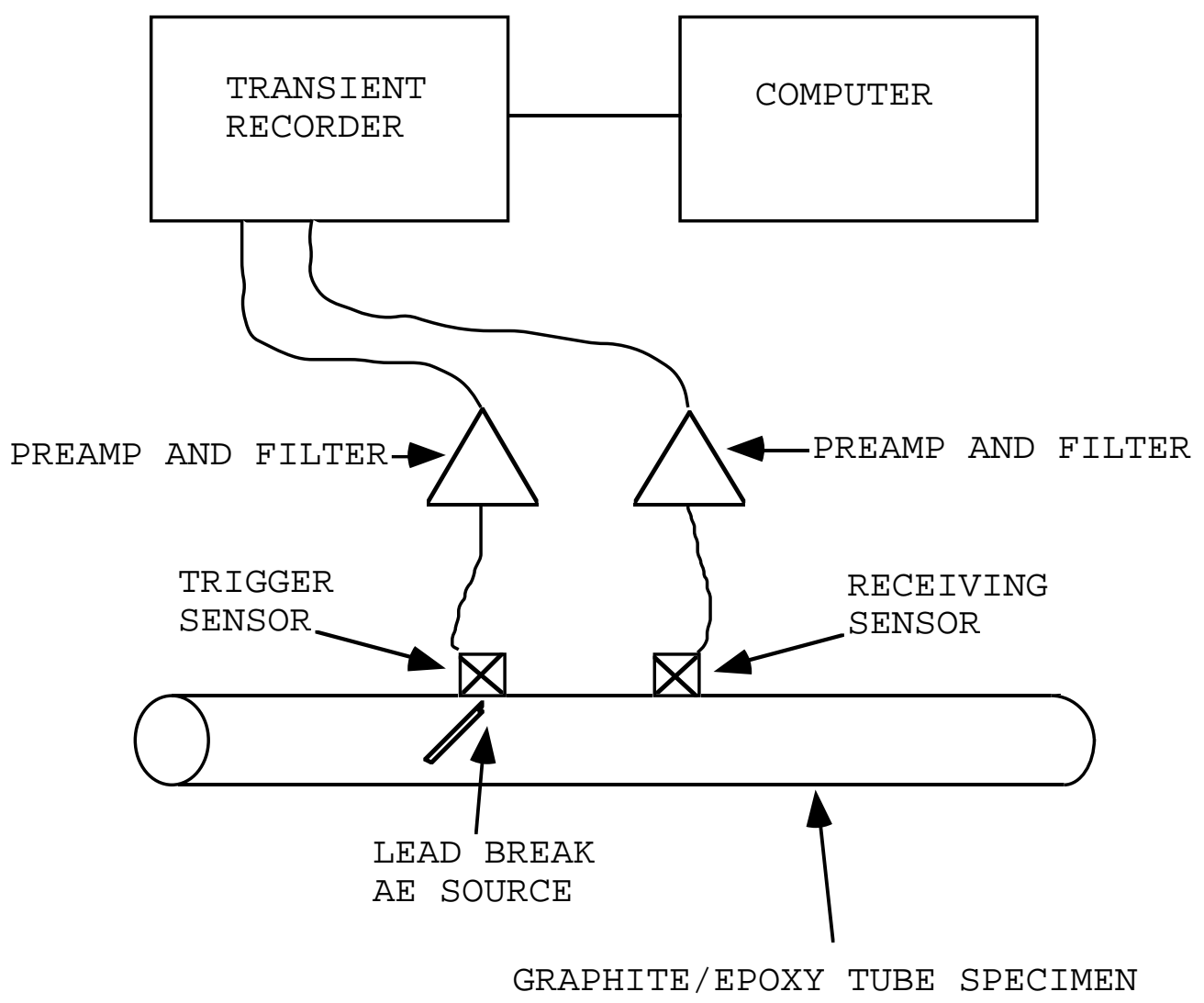

Figure 1. Experimental setup. 


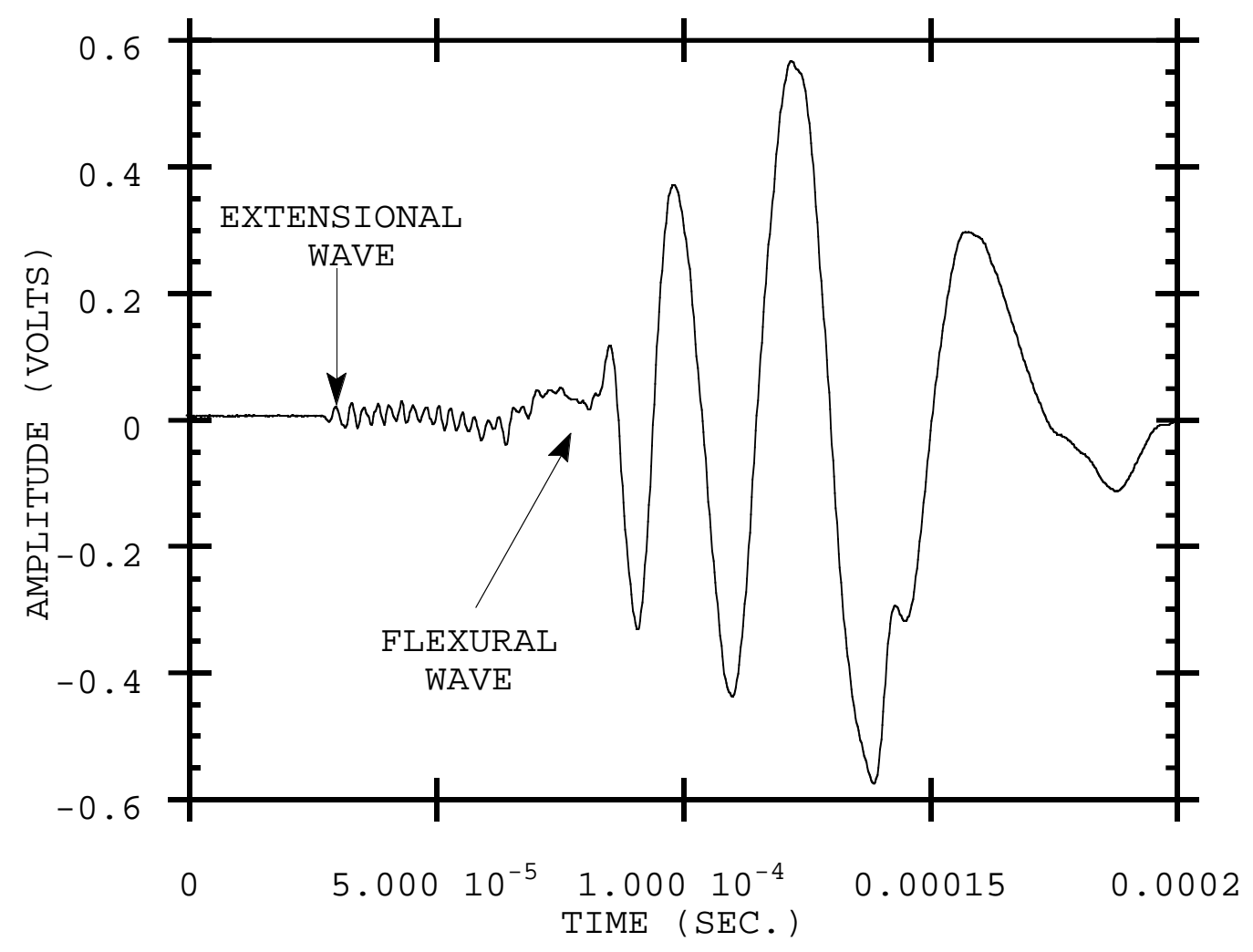

Figure 2. Typical waveform detected on graphite/epoxy tube from lead break source on surface of tube. Source to receive distance is 0.1524 meters. 


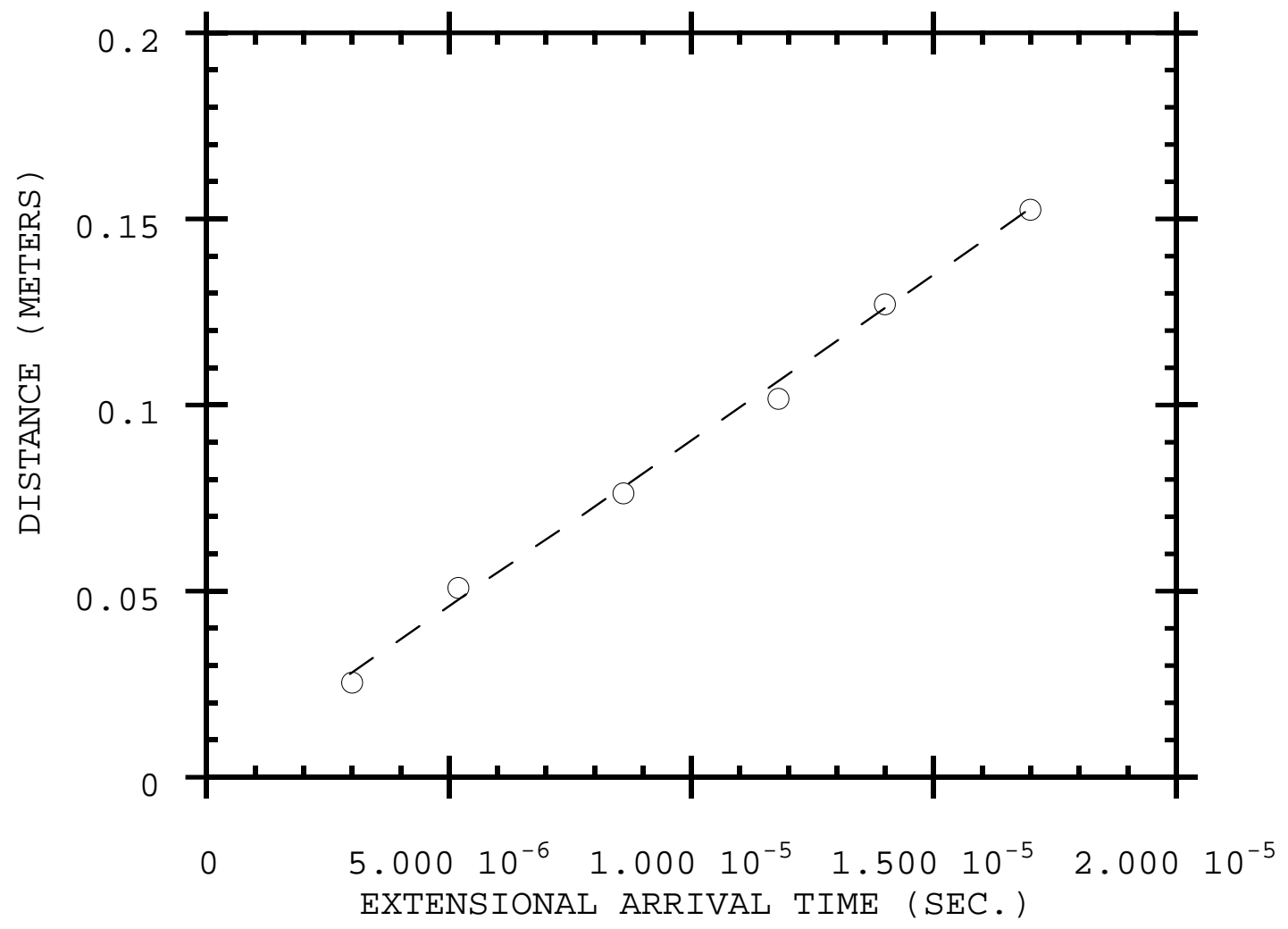

Figure 3. Arrival time of extensional wave versus distance of propagation. Dashed line is least-squares fit used to obtain velocity. 


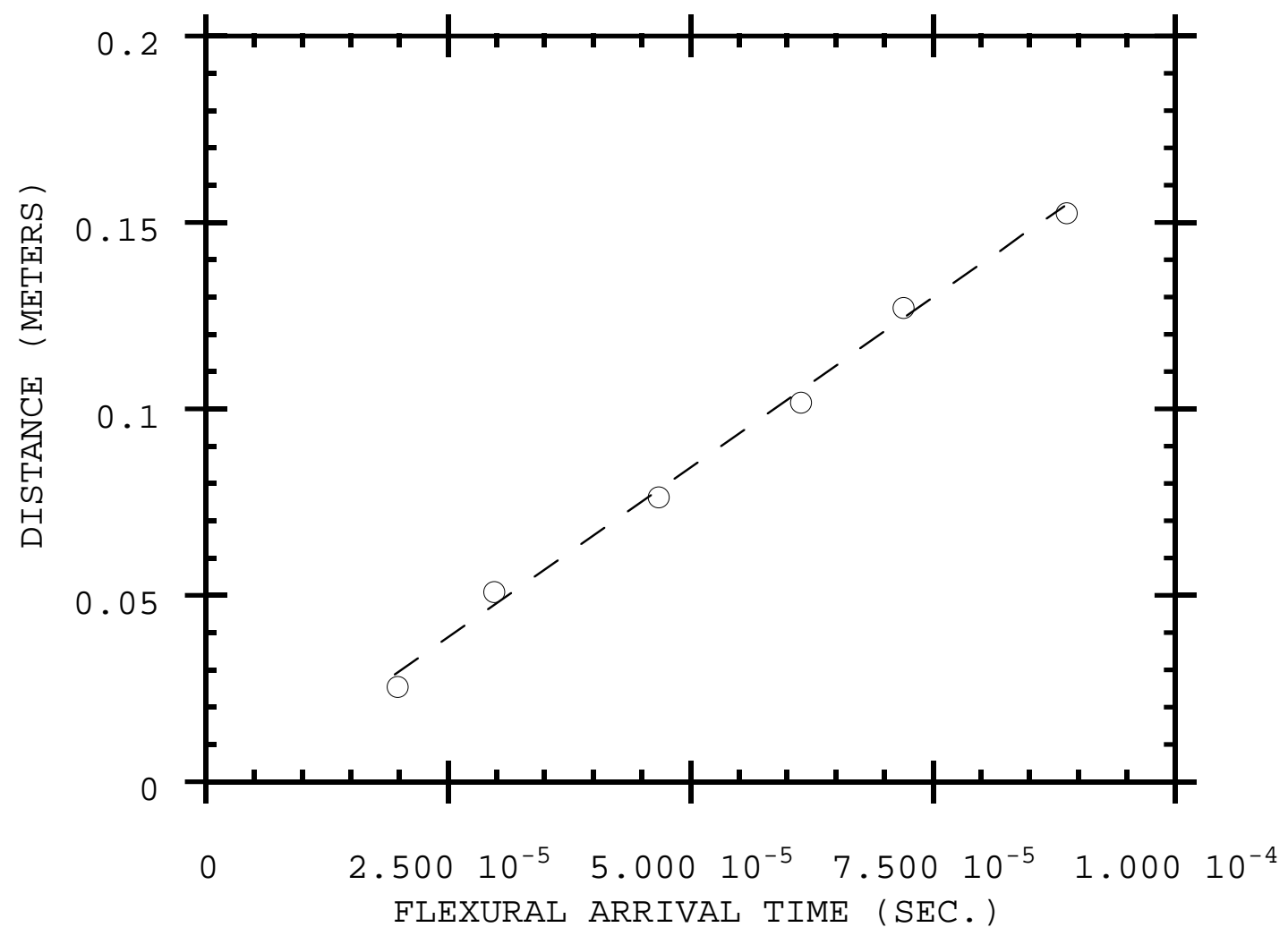

Figure 4. Arrival time of $50 \mathrm{kHz}$ component of flexural wave versus distance of propagation. Dashed line is least-squares fit used to obtain velocity. 


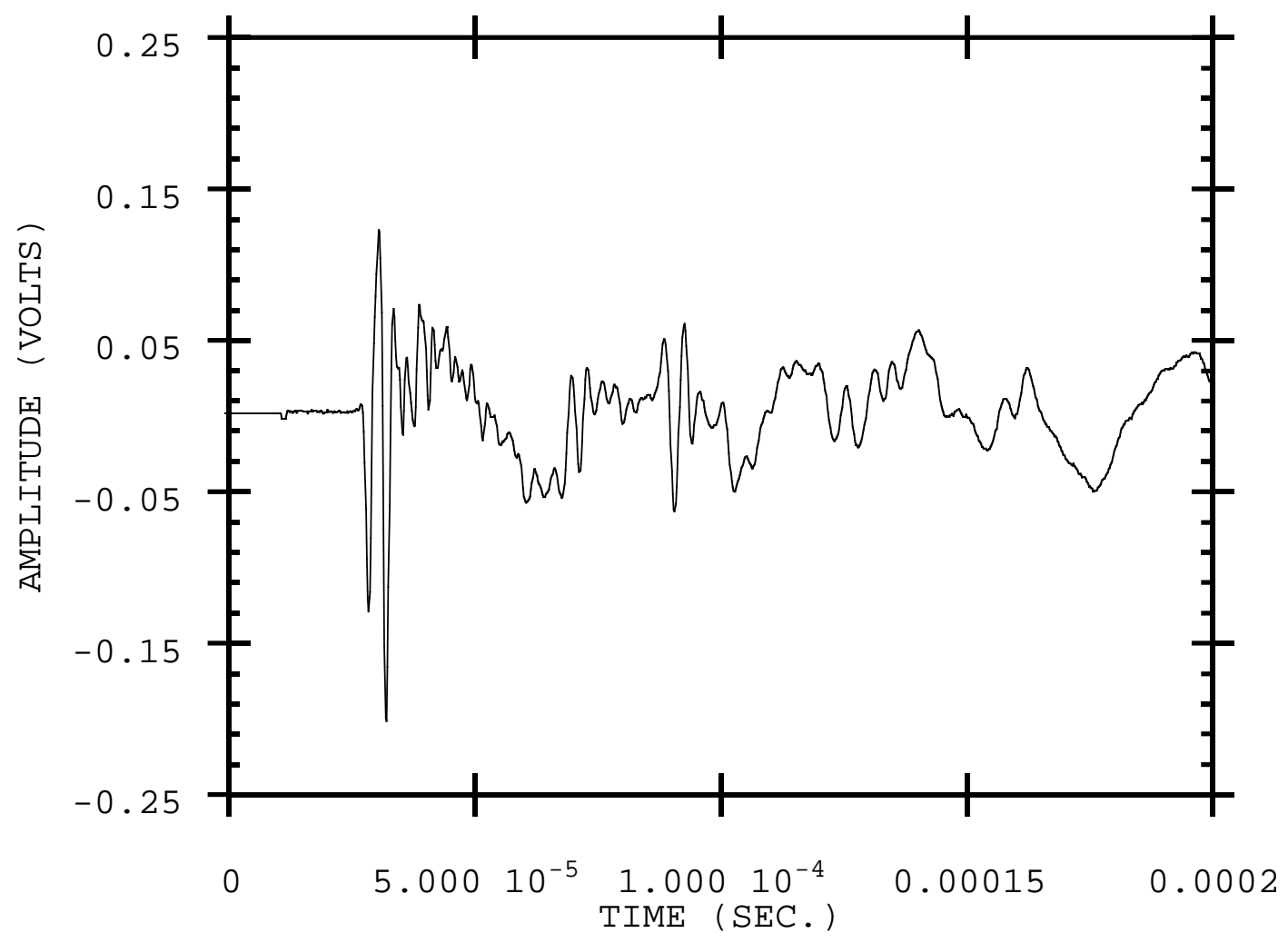

Figure 5. Waveform from lead break on end of tube. Source to receiver distance is 0.1524 meters. 